\title{
Safe use of rocuronium for a post-polio syndrome patient
}

\author{
Kumiko Tanabe $\cdot$ Harumi Takahashi $\cdot$ \\ Hiroki Iida
}

Received: 11 June 2014/ Accepted: 4 August 2014/Published online: 21 August 2014

(C) Japanese Society of Anesthesiologists 2014

To the Editor:

Patients with "post-polio syndrome" can experience recurrent muscle weakness years after acute poliovirus infection [1]. We report a case of a 64-year-old woman who was scheduled for mastectomy. We report the effect of rocuronium, as evaluated by train-of-four (TOF) monitoring of this patient. She had undergone two caesarean sections, under spinal anesthesia with prolongation of hypesthesia and under general anesthesia with delayed awakening, which was speculated to be caused by residual neuromuscular blockade (NMB). She presented with muscle weakness in the lower extremities and the right upper extremity (manual muscle testing 3/5), but spirometry values were normal. After induction of general anesthesia, neuromuscular monitoring was started by use of TOF monitoring of the right adductor pollicis muscle. The TOF ratio was $100 \%$. Rocuronium $(0.6 \mathrm{mg} / \mathrm{kg})$ was used for NMB. Anesthesia was maintained with sevoflurane and remifentanil. The change of TOF ratio is shown in the figure. Because TOF monitoring showed the ratio was $100 \%$ at the end of surgery, NMB was not reversed. She did not report any respiratory complaints.

Electronic supplementary material The online version of this article (doi:10.1007/s00540-014-1906-7) contains supplementary material, which is available to authorized users.

K. Tanabe $(\bowtie) \cdot$ H. Iida

Department of Anesthesiology and Pain Medicine, Gifu

University Graduate School of Medicine, Gifu 501-1194, Japan

e-mail: kumiko-t@m2.gyao.ne.jp

H. Takahashi

Department of Surgery, Gihoku Kousei Hospital, Takatomi,

Japan
Patients with post-polio syndrome are known to be potentially hypersensitive to NMBs, anesthetic agents, and opioids [1]. Because they have fewer motor neurons than normal [1], shorter acting non-depolarizing NMBs are desirable and neuromuscular monitoring is necessary. In our case, it seems likely she did not experience prolongation of the rocuronium effect. Moreover, we did not observe any signs of hypersensitivity to sevoflurane or remifentanil. Prolongation of the effects of these drugs is likely to depend on the extent of paralysis. Recurarization after spontaneous complete recovery does not occur with non-depolarizing NMBs. Although we did not reverse the rocuronium, it might be better to administer sugammadex.

Conflict of interest None.

\section{Reference}

1. Lambert DA, Giannouli E, Schmidt BJ. Postpolio syndrome and anesthesia. Anesthesiology. 2005;103:638-44. 